\title{
Micro-Macro Models for Anisotropic Granular Media
}

\author{
Stefan Luding \\ Particle Technology, DelftChemTech, TUDelft, Julianalaan 136, 2628 BL Delft, The Netherlands, \\ e-mail: s.luding@tnw.tudelft.nl
}

The micro-mechanical behavior of granular materials can be readily modeled with the discrete element method (DEM). From the structure of a static granular solid, we derive the fabric, the stress- and the stiffness tensors in average over a sub-set of interacting particles. Starting from a linear expansion of the interaction potential around static equilibrium, stress and stiffness can be derived from the principle of virtual displacement. The approach includes both normal and tangential forces, and the influence of both on the material properties and the flow behavior is discussed separately. In particular, we present relations for the evolution of both deviatoric fabric and stress with deviatoric deformation.

\section{Introduction}

The macroscopic description of the material behavior of granular materials like sand is one of todays great challenges in material science and physics. Granular are discontinuous, i.e. inhomogeneous, nonlinear, disordered, and anisotropic, on a "microscopic" scale. However, continuum models are applied in large scale industrial design of, e.g., silos.

The rich phenomenology observed in granular matter is due to the changing contact network of the structure formed by the grains, but also due to the inhomogeneous stress distribution in granular assemblies and the corresponding force-networks. There are always large fluctuations of contact forces and a reorganization of the network due to deformation typically leads to a re-structuring of those. When an initially isotropic contact network is deformed, the result is likely to be anisotropic. Bringing all this "microscopic" information all the way up to a macroscopic description via a socalled micro-macro model is one issue of this paper. We do not review the existing literature in this field here, rather we point the readers attention to the books by (Herrmann et al., 1998; Vermeer et al., 2001; Kishino, 2001) and some references by various groups (Chang and Ma, 1991; Babić, 1997; Bagi, 1999; Oda and Iwashita, 2000; Bardet and Vardoulakis, 2001; Suiker et al., 2001; Luding and Herrmann, 2001; Peters and Horner, 2002; Goldhirsch and Goldenberg, 2002; Kruyt, 2003; Luding et al., 2003; Madadi et al., 2004; Luding, 2004; Luding, 2004b) and the references therein.

In the following, a micro-macro formulation based on the virtual displacement ansatz for single contacts is presented as a modular formalism, and the numerical method of discrete element simulations, see e.g. (Oda and Iwashita, 2000; Thornton, 2000; Lätzel et al., 2000), is used to obtain data to compare the macroscopic formulation with. We report the stress-, fabric- and stiffnesstensor component behavior under shear. Furthermore, the anisotropy of the packing and the issue of anisotropic continuum theories is adressed for the set-up used.

2 Micro-macro transition for one contact

For single contacts, the contact force law is reformulated in terms of potential energy density, contact stress, (elastic, reversible) deformation, and stiffness. Since these (tensorial) quantities are associated with single contacts, averages over them can be taken in a variety of ways. Note that the single-contact stresses, for example, do not constitute a macroscopic stress, but they are at the very 
basis of the micro-macro transition, in so far that they contain the "microscopic" contact laws, and can be easily generalized to three dimensions.

The vector that connects the centers of mass $\boldsymbol{r}_{1}$ and $\boldsymbol{r}_{2}$ of two particles, with radius $a$, is the so-called branch vector $\boldsymbol{l}=\boldsymbol{r}_{1}-\boldsymbol{r}_{2}$, with the zero-force (contact) distance $\boldsymbol{l}=|\boldsymbol{l}|=\left(a_{1}+a_{2}\right)$, the particle radii $a_{1}$ and $a_{2}$, and the corresponding unit vector $\hat{\boldsymbol{n}}=\boldsymbol{l} / \boldsymbol{l}$, see also subsection 4.2. The overlap of the two particles is, in normal direction, $\boldsymbol{\Delta}=\boldsymbol{l}-\left(a_{1}+a_{2}\right) \hat{\boldsymbol{n}}=: \boldsymbol{\epsilon}^{n} \cdot \boldsymbol{l}$, corresponding to an elastic (reversible) deformation relative to the touching point. This corresponds to a degenerate tensor of rank two, $\boldsymbol{\epsilon}^{n}=\hat{\boldsymbol{n}}(\hat{\boldsymbol{n}} \cdot \boldsymbol{\epsilon})$, where the dot is the inner (scalar) tensor product that leads to a reduction of the rank by two (no point corresponds to the outer (dyadic) product which leads to a tensor of rank equal to the sum of the two neighboring tensors). The elastic, reversible displacement in tangential direction $\boldsymbol{\vartheta}=: \boldsymbol{\epsilon}^{t} \cdot \boldsymbol{l}$, with $\boldsymbol{\epsilon}^{t}=\hat{\boldsymbol{t}}^{0}\left(\hat{\boldsymbol{t}}^{0} \cdot \boldsymbol{\epsilon}\right)$, (also relative to a stress-free configuration) is irrelevant for perfectly smooth particles, but has to be taken into account for rough surfaces. Thus,

$$
\boldsymbol{\epsilon}=\frac{\Delta}{l} \hat{\boldsymbol{n}} \hat{\boldsymbol{n}}+\frac{\vartheta}{l} \hat{\boldsymbol{t}}^{0} \hat{\boldsymbol{n}}
$$

with $\hat{\boldsymbol{t}}^{0}:=\boldsymbol{\vartheta} /|\boldsymbol{\vartheta}|$, is the state variable conjugate to the stress - at least for the linear force model discussed here - for more details see (Luding, 2004; Luding, 2004b).

The tensor $\boldsymbol{\epsilon}$, which defines a deformation relative to the stress-free configuration, is not necessarily small since overlaps and tangential displacements are not restricted a-priori. In contrast, a virtual, small (infinitesimal) change of the deformation is given by

$$
\delta \boldsymbol{l}=\boldsymbol{l}^{\prime}-\boldsymbol{l}=: \boldsymbol{\varepsilon} \cdot \boldsymbol{l} \approx \delta \boldsymbol{\Delta}+\delta \boldsymbol{\vartheta}=\delta \Delta \hat{\boldsymbol{n}}+\delta \vartheta \hat{\boldsymbol{t}},
$$

where the prime denotes the value after the deformation tensor $\varepsilon$, as discussed in the next subsection, is applied. Note that the vectors $\hat{\boldsymbol{t}}$ and $\hat{\boldsymbol{t}}^{0}$ are not parallel, in general.

\subsection{Small changes of the branch vector}

Given a small, global deformation of the granular packing, the branch vectors will change, but the local deformation is typically different from the global. The local change, $\delta \boldsymbol{l}$, can be split in two components, one parallel to $\hat{\boldsymbol{n}}$, the other one perpendicular to it. The components of the normal change of $\boldsymbol{l}$ are, expressed in index notation where summation over equal indices is implied,

$$
\delta \Delta_{\alpha}=\delta l_{\alpha}^{n}=n_{\alpha} n_{\beta} \varepsilon_{\beta \gamma} l_{\gamma} .
$$

Accordingly, the tangential components are

$$
\delta \vartheta_{\alpha}=\delta l_{\alpha}^{t}=t_{\alpha} t_{\beta} \varepsilon_{\beta \gamma} l_{\gamma},
$$

with the intrinsic definition of the tensor $t_{\alpha} t_{\beta}$ perpendicular to $n_{\alpha} n_{\beta}$. (The tensor $n_{\alpha} n_{\beta}$ is a degenerate, one-dimensional tensor with eigen-direction parallel to $\hat{\boldsymbol{n}}$ and trace unity.)

\subsection{Small changes of the potential energy density}

The potential energy density for one contact with linear normal and tangential springs is:

$$
u:=u_{c}=\frac{1}{2 V_{c}}\left(k \boldsymbol{\Delta}^{2}+k^{t} \boldsymbol{\vartheta}^{2}\right),
$$

where $k$ and $k^{t}$ are the spring stiffness in normal and tangential direction, respectively (the prefactors of the quadratic term in a series expansion of the interaction potential), and the volume $V_{c}$ is not specified here, see (Luding, 2004), since it disappears during averaging, in many cases. A small displacement of one pair of particles leads to the change in potential energy density

$$
\delta u=\delta u^{n}+\delta u^{t} \approx \frac{1}{V_{c}}\left(k \Delta \delta l^{n}+k^{t} \vartheta_{\alpha} \delta l_{\alpha}^{t}\right) \approx \frac{1}{V_{c}} \boldsymbol{f}^{*} \cdot \boldsymbol{\varepsilon} \cdot \boldsymbol{l},
$$

with the actual force $\boldsymbol{f}=k \boldsymbol{\Delta}+k^{t} \boldsymbol{\vartheta}$, the force after displacement $\boldsymbol{f}^{\prime}=\boldsymbol{f}+\delta \boldsymbol{f}$, and the mean $f^{*}=\left(f+f^{\prime}\right) / 2$. (The asterisk is dropped in the following for the sake of simplicity implying: $f \approx f^{*}$ ). Note the nice symmetry of the problem with respect to an exchange of the present configuration (unprimed) and the deformed configuration (primed). 


\subsection{The stress tensor from a static contact}

From the potential energy density, we obtain the transposed stress from the response to a virtual deformation by differentiation of $u$ with respect to the deformation tensor components

$$
\sigma_{\beta \alpha}=\frac{\partial u}{\partial \varepsilon_{\alpha \beta}}=\frac{1}{V_{c}} f_{\alpha} l_{\beta} .
$$

For the result in Eq. (7), the partial derivative of the displacement vector with respect to the deformation tensor was replaced by the branch vector component and the identity tensors $1_{\alpha a} 1_{\beta b}$; higher order terms in Eq. (6) were neglected. Both $\boldsymbol{l}$ and $\boldsymbol{\Delta}$ are parallel to $\hat{\boldsymbol{n}}$ and $\boldsymbol{\vartheta}$ is parallel to $\hat{\boldsymbol{t}}^{0}$, so that one can rewrite the stress tensor

$$
\sigma_{\alpha \beta}=\frac{k l \Delta}{V_{c}} n_{\alpha} n_{\beta}+\frac{k^{t} l \vartheta}{V_{c}} n_{\alpha} t_{\beta}^{0}
$$

and the stress increment tensor

$$
\delta \sigma_{\alpha \beta} \approx \frac{k l \delta \Delta}{V_{c}} n_{\alpha} n_{\beta}+\frac{k^{t} l \delta \vartheta}{V_{c}} n_{\alpha} t_{\beta}
$$

with $\Delta=|\boldsymbol{\Delta}|, \delta \boldsymbol{\Delta}=|\delta \boldsymbol{\Delta}|, \vartheta=|\boldsymbol{\vartheta}|$, and $\delta \vartheta=|\delta \vartheta|$. Note that the dyadic product of the normal vectors $n_{\alpha} n_{\beta}$ is symmetric (and degenerate one-dimensional) by definition. In $2 \mathrm{D}$, one has $n_{\alpha} t_{\beta}^{0}= \pm n_{\alpha} t_{\beta}$, where the tensor objects are typically non-symmetric and traceless. The stress relations above are similar to those obtained earlier in the literature for many particle contacts (Bardet and Vardoulakis, 2001; Lätzel et al., 2000; Rothenburg and Selvadurai, 1981; Bathurst and Rothenburg, 1988; Bardet and Proubet, 1991; Bagi, 1996; Liao and Chang, 1997; Kruyt and Rothenburg, 1998; Kruyt and Rothenburg, 2001; Ball and Blumenfeld, 2002; Kruyt, 2003).

\subsection{The stiffness tensor from a static contact}

The partial derivative of the stress tensor with respect to the deformation leads to the single-contact stiffness

$$
C_{\alpha \beta \gamma \phi}=\frac{\partial \sigma_{\alpha \beta}}{\partial \varepsilon_{\phi \gamma}}=\frac{l^{2}}{V_{c}}\left(k n_{\alpha} n_{\beta} n_{\gamma} n_{\phi}+k^{t} n_{\alpha} t_{\beta} n_{\gamma} t_{\phi}\right),
$$

where the changes of the deformation in normal and tangential direction were used. The additional derivative which should occur in Eq. (10) leads to terms proportional to $\Delta / l$, which are neglected in the following, since the overlap is typically much smaller than the distance between the particle centers.

Note that the stiffness tensor in Eq. (10) is similar to the results in the literature, see e.g. (Liao and Chang, 1997; Kruyt and Rothenburg, 1998), but here the contribution of a single contact only is given. In the next section, the relations are provided for many particles inside larger averaging volumes.

\section{Volume averaging}

In this section, averages over the single-contact tensors from the previous section are taken, for the sake of completeness, to be compared to the previous literature, see e.g. (Rothenburg and Selvadurai, 1981; Liao and Chang, 1997; Kruyt and Rothenburg, 1998; Lätzel et al., 2000). Note that we focus here on those quantities that can be obtained from static snapshots and do not rely on (real) displacements, see (Luding, 2004; Luding, 2004b) for more details.

For the sake of simplicity, the simplest averaging approach is used here, i.e. a contact is taken into account if the corresponding particle center lies within the averaging volume. This corresponds to a pre-averaging over single particles and then subsequent averaging over the particles in the volume. Cast into an equation this reads

$$
Q=\langle Q\rangle=\frac{1}{V} \sum_{p \in V} V^{p} Q^{p},
$$

where $Q$ is the quantity to be averaged and $Q^{p}=\left(1 / V^{p}\right) \sum_{c=1}^{C^{p}} V_{c} Q^{c}$ is the pre-averaged particle quantity with the contact quantity $Q^{c}$. Here, the sub-script $p \in V$ denotes the particle-in-volume averaging procedure. As the simplest example, the volume fraction $\nu$ is thus obtained from $Q^{p}=1$. 


\subsection{The Fabric Tensor}

For one particle with $C^{p}$ contacts, the fabric tensor is defined as the sum, over all contacts, of the dyadic product formed by the normal vectors:

$$
F_{\alpha \beta}^{p}=\sum_{c=1}^{C^{p}} n_{\alpha} n_{\beta},
$$

with the trace $\operatorname{tr} \boldsymbol{F}^{p}=F_{\gamma \gamma}^{p}=C^{p}$. In a large volume, with some distribution of particle radii, the relation between trace of fabric, density and average contact number $C$ is according to (Madadi et al., 2004): $F_{\alpha \alpha}=g_{2} \nu C$, with the average fabric

$$
F_{\alpha \beta}=\frac{1}{V} \sum_{p \in V} V^{p} \sum_{c=1}^{C^{p}} n_{\alpha} n_{\beta}
$$

and the correction factor

$$
g_{2} \approx 1+\frac{\sqrt{3}}{\pi}\left(\frac{\overline{a^{3}}}{\bar{a} \overline{a^{2}}}-1\right)
$$

dependent on the first three moments of the size distribution $\overline{a^{k}}$ (with $k=1,2,3$ ), see the study by (Madadi et al., 2004). In brief, $g_{2}$ corrects for the fact that the coordination number of different sized particles is proportional to their surface area, so that a monodisperse packing has $g_{2}=1$, whereas a polydisperse packing has $g_{2}>1$ with magnitude increasing with the width of the size distribution. Thus, a polydisperse packing has a higher contact number density than a monodisperse system of comparable density. It was shown recently that the correction, as tested for frictionless systems (Madadi et al., 2004), is also relevant for frictional packings (Luding, 2004; Luding, 2004b).

\subsection{The Stress Tensor}

In the averaging volume $V$, one obtains the approximate (averaged) macroscopic stress from Eqs. (8) and (11) so that:

$$
\sigma_{\alpha \beta}=\frac{1}{V} \sum_{p \in V} \sum_{c=1}^{C^{p}} l_{\alpha}^{c} f_{\beta}^{c}
$$

with the center to contact branch vector $\boldsymbol{l}^{c}$. The particle volumes (and the arbitrary averaging volumes introduced for the single-particle relations) cancel due to the volume weight in Eq. (11).

\subsection{The stiffness tensor}

The stiffness tensor for spherical (disk) particles with branch vectors from the center to the contactpartner $l=l^{c}$, and identical spring constants $k=k^{c}$ and $k^{t}=\left(k^{t}\right)^{c}$, is equivalently:

$$
C_{\alpha \beta \gamma \phi}=\frac{1}{V} \sum_{p \in V}\left(k \sum_{c=1}^{C}\left(l^{2} / 2\right) n_{\alpha}^{c} n_{\beta}^{c} n_{\gamma}^{c} n_{\phi}^{c}+k^{t} \sum_{c=1}^{C}\left(l^{2} / 2\right) n_{\alpha}^{c} t_{\beta}^{c} n_{\gamma}^{c} t_{\phi}^{c}\right),
$$

where the two contributions from normal and tangential springs will be examined separately below. Note the factor $1 / 2$ for the contribution from each of the two contacting particles, which could be replaced by $a / l$ or $2 a^{2} / l^{2}$ for mono-sized particles. For the rather narrow size-distribution used below, we selected empirically the simplest choice. Again this result can already be found in the literature (Liao and Chang, 1997; Kruyt and Rothenburg, 1998) in similar form, however, we provide it here again, for the sake of completeness, in our nomenclature. More details and results on periodic lattices will be presented in a forthcoming paper (Luding, 2004b). 


\subsection{Model System}

The discrete element model (DEM) (Herrmann et al., 1998; Vermeer et al., 2001; Cundall and Strack, 1979; Bashir and Goddard, 1991; van Baars, 1996; Oda and Iwashita, 2000; Thornton, 2000; Thornton and Antony, 2000; Oda and Iwashita, 2000; Kruyt and Rothenburg, 2001) is briefly introduced in this section, for more details see the papers (Luding and Herrmann, 2001; Luding et al., 2003; Madadi et al., 2004; Luding, 2004; Luding, 2004b)

The "experiment" chosen is the biaxial box set-up, where the left and bottom walls are fixed, and stress- or strain-controlled deformation is applied to the side- and top-wall, respectively. In the first case a wall is subject to a pre-defined pressure, in the second case, the wall is subject to a pre-defined strain. In a typical "experiment", the top wall is slowly shifted downwards, while the right wall moves stress controlled, dependent on the force $F(t)$ exerted on it by the material in the box. The (strain-controlled) position of the top wall is a cosine function that is chosen in order to allow for a smooth start-up and finish of the motion so that shocks and inertia effects are reduced, however, the shape of the function is arbitrary as long as it is smooth and the deformation is slow.

\subsection{Discrete Particle Model}

The elementary units of granular materials are mesoscopic grains which deform under stress. Since the realistic modeling of the deformations of the particles is much too complicated, we relate the normal interaction force to the overlap $\Delta$ of two particles. Note that the evaluation of the interparticle forces based on the overlap may not be sufficient to account for the inhomogeneous stress distribution inside the particles. Consequently, our results presented below are of the same quality as the simple assumptions about the force-overlap relation.

If all forces $\boldsymbol{f}_{i}$ acting on the particle $i$, either from other particles, from boundaries or from external forces, are known, the problem is reduced to the integration of Newton's equations of motion for the translational and rotational degrees of freedom

$$
m_{i} \frac{\mathrm{d}^{2}}{\mathrm{~d} t^{2}} \boldsymbol{r}_{i}=\boldsymbol{f}_{i}, \text { and } I_{i} \frac{\mathrm{d}^{2}}{\mathrm{~d} t^{2}} \boldsymbol{\varphi}_{i}=\boldsymbol{t}_{i}
$$

with the mass $m_{i}$ of particle $i$, its position $\boldsymbol{r}_{i}$ the total force $\boldsymbol{f}_{i}=\sum_{c} \boldsymbol{f}_{i}^{c}$ acting on it due to contacts with other particles or with the walls, its moment of inertia $I_{i}$, its angular velocity $\boldsymbol{\omega}_{i}=\mathrm{d} \boldsymbol{\varphi}_{i} / \mathrm{d} t$ and the total torque $\boldsymbol{t}_{i}=\sum_{c} \boldsymbol{l}_{i}^{c} \times \boldsymbol{f}_{i}^{c}$.

Two particles $i$ and $j$ interact only if they are in contact so that their overlap $\Delta:=-\hat{\boldsymbol{n}} \cdot \boldsymbol{\Delta}$ is positive. The force on particle $i$, from particle $j$ can be decomposed into a normal and a tangential part, where the simplest normal force is a linear spring and a linear dashpot

$$
f_{i}^{n}=k \Delta+\gamma_{0} \dot{\Delta}
$$

with spring constant $k$ and some damping coefficient $\gamma_{0}$. The half-period of a vibration around the equilibrium position can be computed, and one obtains a typical response time $t_{c}=\pi / \omega$, with $\omega=$ $\sqrt{\left(k / m_{i j}\right)-\eta_{0}^{2}}$, the eigenfrequency of the contact, the reduced mass $m_{i j}=m_{i} m_{j} /\left(m_{i}+m_{j}\right)$, and the rescaled damping coefficient $\eta_{0}=\gamma_{0} /\left(2 m_{i j}\right)$. The energy dissipation during a collision, as caused by the dashpot, leads to a restitution coefficient $r=-v_{n}^{\prime} / v_{n}=\exp \left(-\eta_{0} t_{c}\right)$, where the prime denotes the normal velocity after a collision. Details about the implementation of alternative contact models and tangential contact force laws for $\boldsymbol{f}_{i}^{t}$ can be found in (Luding et al., 2003; Luding, 2004; Luding, 2004b).

\subsection{Parameters and initial configuration}

The system examined in the following contains $N=1950$ particles with radii $a_{i}$ randomly drawn from a homogeneous distribution between $a_{\min }=0.510^{-3} \mathrm{~m}$ and $a_{\max }=1.510^{-3} \mathrm{~m}$. The masses of the cylindrical particles with height $h=2.010^{-4} \mathrm{~m}$ are $m_{i}=\rho \pi h a_{i}^{2}$, with the density $\rho=$ $2.010^{3} \mathrm{~kg} \mathrm{~m}^{-3}$. The total mass of the particles in the system is thus $M \approx 0.0026 \mathrm{~kg}$ with the typical reduced mass of a pair of particles with mean radius, $m_{12} \approx 0.6710^{-6} \mathrm{~kg}$. The wall properties are $m_{\mathrm{w}}=10^{-4} \mathrm{~kg}$ and $\gamma_{\mathrm{w}}=2 \mathrm{~kg} \mathrm{~s}^{-1}$. If not explicitly mentioned, the material parameters are $k=10^{5} \mathrm{Nm}^{-1}, \gamma_{0}=\gamma_{t}=0.02 \mathrm{~kg} \mathrm{~s}^{-1}$, and $\gamma_{\mathrm{b}}=10^{-5} \mathrm{~kg} \mathrm{~s}^{-1}, \mu=0.5$, and $k_{t} / k=0.2$. This 
leads to a typical contact duration $t_{c}=0.8210^{-5} \mathrm{~s}$ and a restitution coefficient of $r=0.89$, with the integration time-step used $\delta t_{M D}=0.210^{-6} \mathrm{~s}$. The choice of parameters is rather arbitrary, however, the finding below that the stiffness tensor scales with the spring contstant rectifies it a-posteriori. Additional simulations (not shown here) also confirm this statement. Note that the choice of the stiffness and a possible non-linear force law is more important for dynamic systems for, e.g., sound propagation than for the quasi-static system presented here.

Initially, the particles are randomly distributed in a huge box, with rather low overall density. Then the box is compressed with isotropic pressure $p=p_{\mathrm{x}}=p_{\mathrm{z}}$, in order to achieve an initial condition as isotropic as feasible; there is remainig anisotropy of the order of a few per-cent in some situations, however. This configuration is relaxed until the kinetic energy is several orders of magnitude smaller than the potential contact energy. Starting from the relaxed, isotropic initial configuration, the strain is applied to the top wall and the response of the system is examined, while the side wall is still pressure controlled.

\subsection{Averaged Quantities}

In the following, simulation results are presented for various side pressures $p$. Averages are performed such that parts of the system close to the walls are disregarded in order to avoid boundary effects. This means, that the averaging volume is only 64 per-cent of the total volume. A particle contact is taken into account for the average if the corresponding particle-center lies within the averaging volume $V$.

\subsubsection{Density and volumetric strain}

The first quantity of interest is the density (volume fraction) $\nu$ and, related to it, the volumetric strain $\epsilon_{V}=\Delta V / V$. From the averaged data, we evidence initial compression for small deformations and for larger side pressures, see Fig. 1. This initial regime follows strong dilation, for all pressures, until a quasi-steady-state critical flow regime is reached, where the density is almost constant besides a weak tendency towards further dilation.
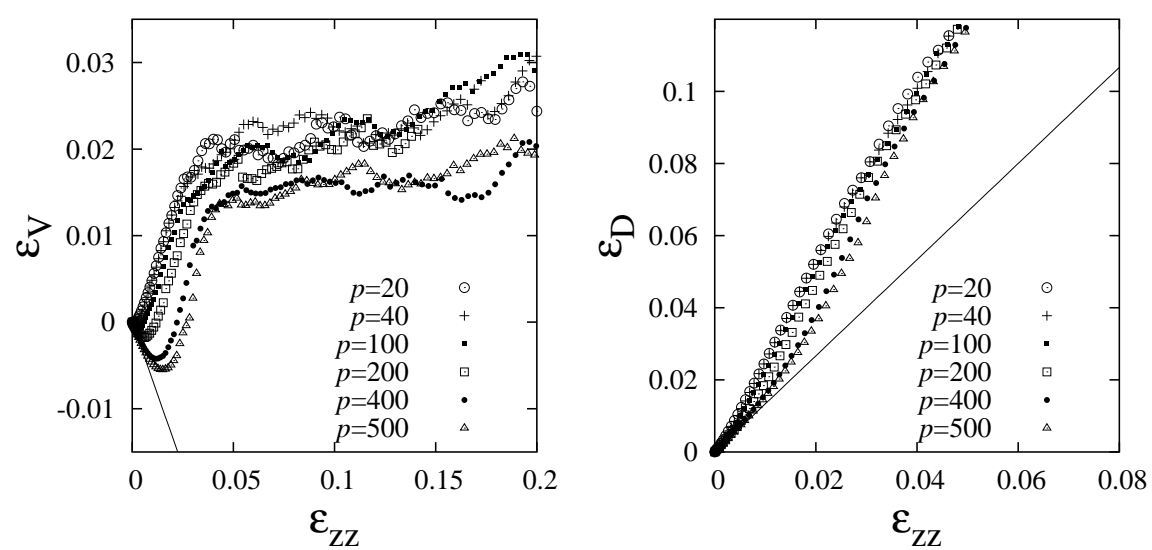

Figure 1: (Left) Volumetric strain $\varepsilon_{V}$ - negative values mean compression whereas positive values correspond to dilation - and (Right) Deviatoric strain $\varepsilon_{D}=\varepsilon_{z z}-\varepsilon_{x x}$, both plotted against the vertical strain $\varepsilon_{z z}$. The lines correspond to $-(2 / 3) \varepsilon_{z z}$ and $(4 / 3) \varepsilon_{z z}$, respectively.

The second quantity of interest is the deviatoric strain $\varepsilon_{D}$ that quantifies the amount of shear the medium undergoes in addition - and independently - of the compression/dilatancy. Initially, one has $\varepsilon_{D} \approx-2 \varepsilon_{V}$, but the deviation is stronger and begins earlier with decreasing side pressure.

An initially dilute granular medium (weak confining pressure) thus shows dilation from the beginning, whereas a denser granular material (strong confining pressure) can be compressed even further by the relatively strong external forces before dilation starts. The range of density changes is about 0.02 in volume fraction and spans $2-3 \%$ changes in volumetric strain, for the parameters used here. The material undergoes continuous shear - due to the boundary conditions chosen where the shear is stronger and grows faster for smaller confining stress $p$, i.e. for larger $p$, the 
material is more resistant to shear.

\subsubsection{Fabric Tensor}

The fabric tensor is computed according to Eq. (13), and its isotropic and deviatoric contributions are displayed in Fig. 2. The isotropic contribution is scaled by the prediction from (Madadi et al., 2004), and the deviation from the prediction is between one to three percent, where the larger side pressure data are in better agreement (smaller deviation). Note that the correction due to the factor $g_{2}$ corresponds to about nine per-cent, and that the data are taken in the presence of friction, in contrast to the simulations by (Madadi et al., 2004).

The anisotropy of the granular packing is quantified by the deviatoric fabric, as displayed in its scaled form in Fig. 2. The anisotropy is initially of the order of a few percent - thus the initial configurations are not perfectly isotropic. With increasing deviatoric deformation, the anisotropy grows, reaches a maximum and then saturates on a lower level in the critical state flow regime. The relative anisotropy is stronger and grows faster for smaller side pressure, whereas the nonscaled fabric deviator, astonishingly, grows to values around $0.56 \pm 0.03$, independently of the side pressure (data not shown here, see (Luding, 2004) for details).
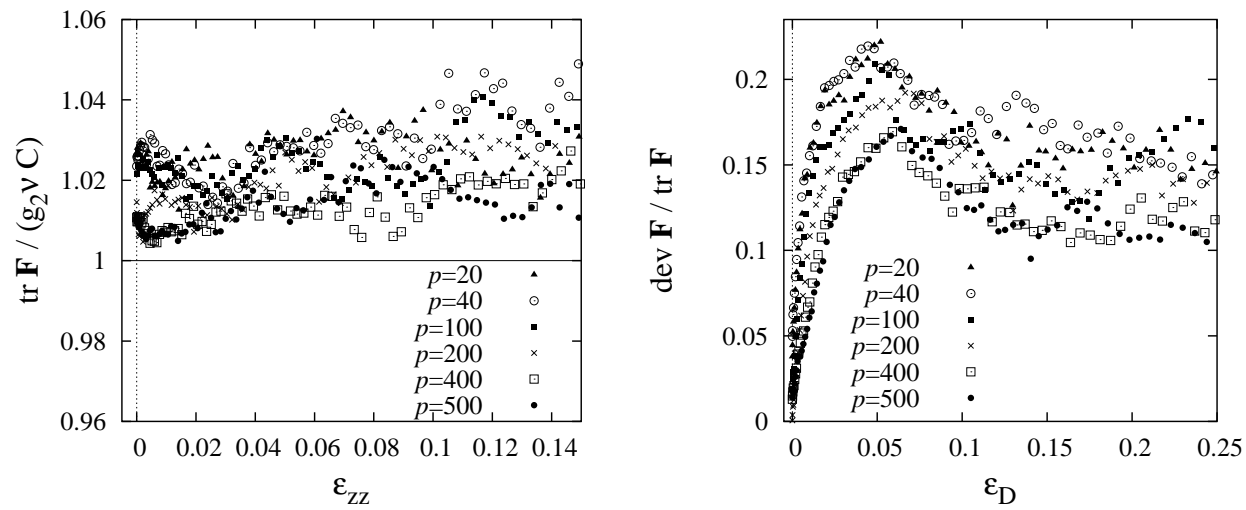

Figure 2: (Left) Quality factor for the trace of the fabric tensor scaled by the analytical prediction $g_{2} \nu C$ from (Madadi et al., 2004), for different pressures $p$, as function of vertical deformation. (Right) Deviatoric fraction of the fabric tensor from the same simulations plotted against the deviatoric deformation.

Using the definition $f_{D}:=\operatorname{dev} \boldsymbol{F} / \operatorname{tr} \boldsymbol{F}$, one finds that the deviatoric fabric approaches a maximal value close to $f_{D}^{\max }(\nu, p) \operatorname{tr} \boldsymbol{F} \approx 0.6$, see (Luding, 2004), a function of, at least, density and confining stress, which varies only very weakly with $p$. The question examined in the following is: How does the fabric approach the "yield-limit" $f_{D} \leq f_{D}^{\max }(\nu, p)$. Based on the numerical simulations, and related to experimental observations, see section 5.2.3 in the paper by (Calvetti et al., 1997), we propose the following differential equation, which describes an exponential approach of the deviatoric fabric to its limit value:

$$
\frac{\partial f_{D}}{\partial \varepsilon_{D}}=\beta_{F}\left(f_{D}^{\max }-f_{D}\right),
$$

where $\beta_{F}=\beta_{F}(p)$ is a material parameter that depends only weakly on $p\left(\beta_{F}(p=200) \approx\right.$ 82 ), and the deviatoric deformation $\varepsilon_{D}=\varepsilon_{z z}-\varepsilon_{x x}$ is introduced. This equation is solved by $1-\left(f_{D} / f_{D}^{\max }\right)=\exp \left(-\beta_{F} \varepsilon_{D}\right)$, in agreement with the simulation data for $\varepsilon_{D} \leq 0.03$, with an error margin of about five per-cent. When the maximal anisotropy is reached, the behavior changes possibly due to shear band localization, and $f_{D}^{\max }$ is reduced to its critical state value $f_{D}^{\text {crit }}$. Remarkable is here that both $f_{D}^{\max } \operatorname{tr} \boldsymbol{F}$ and $f_{D}^{\text {crit }} \operatorname{tr} \boldsymbol{F}$ are only very weakly dependent on $p$.

The formulation of a more general constitutive law for arbitrary orientation of the deformation direction relative to the direction of the fabric eigen-values is far from the scope of this paper. 


\subsubsection{Stress tensor}

The behavior of the stress is displayed in Fig. 3, where the isotropic stress (1/2)tr $\sigma$ is plotted in units of $p$, and the deviatoric fraction is plotted in units of the normal stress. Note that the tangential forces do not contribute to the isotropic stress here since its entries compensate. From Fig. 3, we evidence that both normal contributions, the non-dimensional trace and the non-dimensional deviator behave similarly, independent of the side pressure: Starting from an initial value, a maximum is approached - where the maximum is only weakly dependen on $p$ - the approach is faster for lower $p$. After the maximum is reached, the stresses approaches a smaller value in the critical state flow regime.
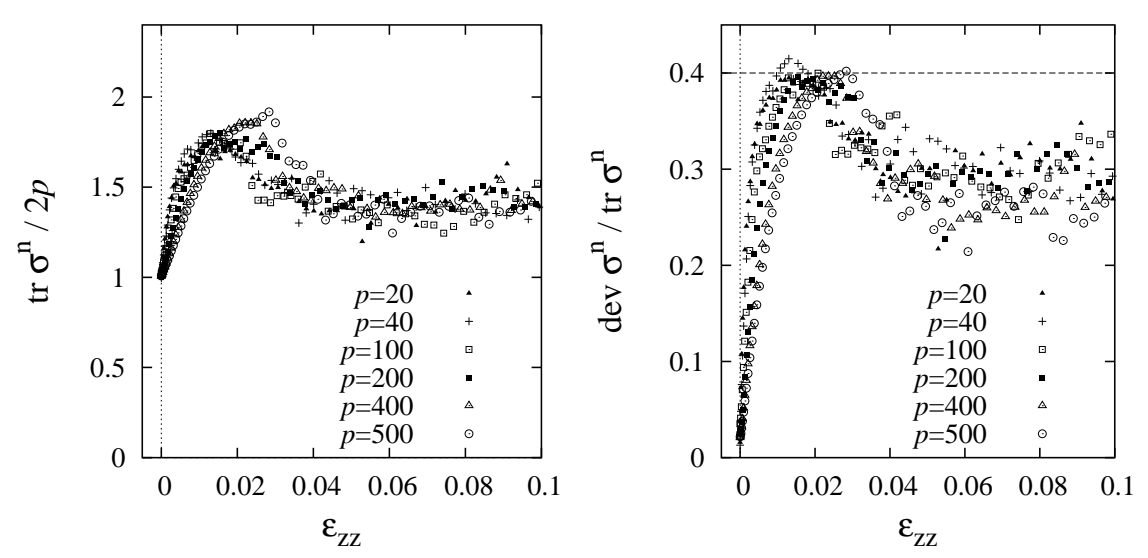

Figure 3: Non-dimensional stress tensor contributions for different $p$. The isotropic (Left) and the deviatoric fractions (Right) are displayed as functions of the vertical displacement.

Using the definitions $s_{V}:=\operatorname{tr} \sigma /(2 p)-1$ and $s_{D}:=\operatorname{dev} \sigma / \operatorname{tr} \sigma$, the maximal (non-dimensional) isotropic and deviatoric stresses are $s_{V}^{\max } \approx 0.8 \pm 0.1$ and $s_{D}^{\max } \approx 0.4 \pm 0.02$, respectively, with a rather large error margin. The corresponding values at critical state flow are $s_{V}^{c} \approx 1.4 \pm 0.1$ and $s_{D}^{c} \approx 0.29 \pm 0.04$.

The isotropic stress $\sigma_{V}=\operatorname{tr} \sigma / 2$ (data not shown here) is appropriately described (for small anisotropy) by an empirical fit $\sigma_{V}=\sigma_{h}\left(\nu-\nu_{h}\right)^{h}$, with the values $\sigma_{h} / k_{n}=1.23 \pm 0.18, \nu_{h}=$ $0.850 \pm 0.001$, and $h=1.26 \pm 0.06$. Note the rather large uncertainty and also the fact that, e.g., $\nu_{h}$ is a function of the history of the sample. For densities smaller than $\nu_{h}$, there is no stable static configuration observed.
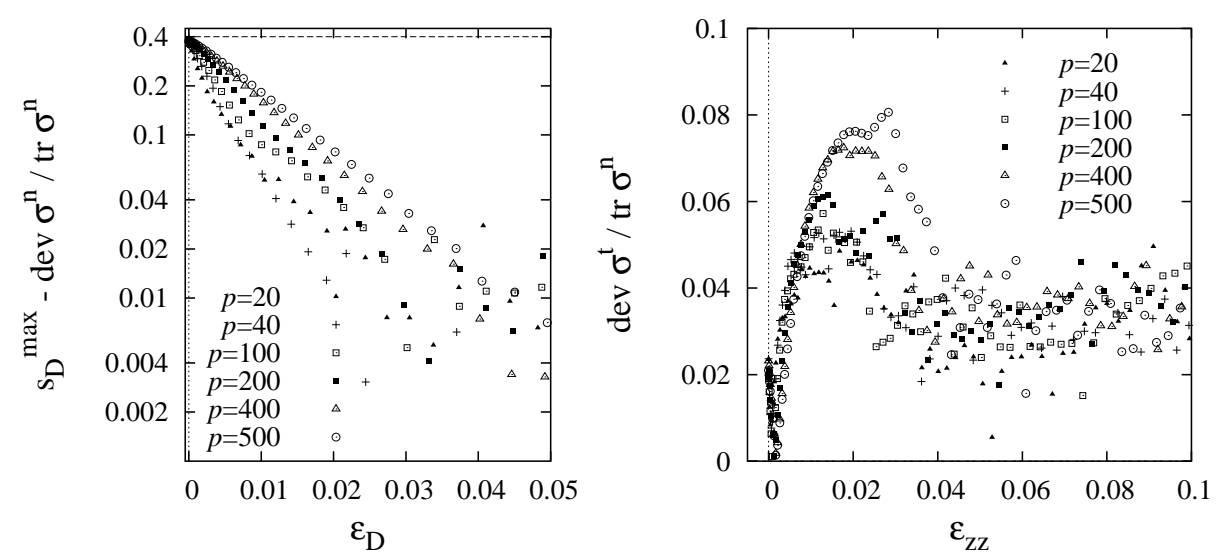

Figure 4: Non-dimensional deviatoric stresses for different $p$. (Left) The approach to $s_{D}^{\max }$ is shown in semi-logarithmic scale. (Right) The tangential contribution to the stress is displayed as function of the vertical deformation, scaled by the isotropic stress. 
The evolution of the deviatoric stress fraction, $s_{D}$, as function of $\varepsilon_{D}$ is displayed in Fig. 4, for small $\varepsilon_{D}$, in semi-logarithmic scale, indicating an exponential approach towards the maximum, which is described by the differential equation

$$
\frac{\partial s_{D}}{\partial \varepsilon_{D}}=\beta_{s}\left(s_{D}^{\max }-s_{D}\right)
$$

where $\beta_{s}=\beta_{s}(p)$ is a material parameter decaying with increasing $p$ roughly as $\beta_{s} \approx p^{-1 / 2}$.

The deviatoric stress from the tangential forces shows a maximum that is increasing with $p$, so that friction is more important for larger side pressure. For $p=20$ and 500 , the peak stress due to frictional forces is about $1 / 10$ and $1 / 5$ of the normal stress contribution, respectively.

\subsubsection{Stiffness tensor}

Given an arbitrary deformation, the stiffness tensor relates the stress changes to the deformation

$$
\delta \sigma_{\alpha \beta}=C_{\alpha \beta \gamma \phi} \varepsilon_{\gamma \phi}+\delta \sigma_{\alpha \beta}^{\text {struct. }},
$$

where the first term corresponds to the elastic (reversible) structural anisotropy, and the second term contains the stress changes due to a change in structure. The stiffness tensor entries $C_{\alpha \beta \gamma \phi}$ were discussed in detail in (Luding, 2004), where it was concluded that there are typically only three different moduli $C_{1}:=C_{x x x x}, G:=C_{x x z z}=C_{z z x x}$, and $C_{2}:=C_{z z z z}$ due to normal forces, in the coordinate system of the biaxial box. Tensor entries with an odd number of indices are practically zero. The stiffness entries due to the tangential forces could be related to these three moduli. In the following, we focus on the first term in Eq. (21), and rewrite it in terms of $\sigma_{V}$, $\sigma_{D}, \varepsilon_{V}$, and $\varepsilon_{D}$, where the indices $V$ and $D$ denote the isotropic and the deviatoric contributions, respectively. In the biaxial box system, where the eigensystem of the tensors is oriented according to the wall geometry, a scalar formulation is possible for the isotropic stress:

$$
\delta \sigma_{V}=\delta \sigma_{\alpha \alpha} / 2=E\left(\varepsilon_{V}+\frac{A}{E} \varepsilon_{D}\right)+\delta \sigma_{\alpha \alpha}^{\text {struct. }}
$$

with $\varepsilon_{V}=(1 / 2)\left(\varepsilon_{x x}+\varepsilon_{z z}\right), \varepsilon_{D}=(1 / 2)\left(\varepsilon_{z z}-\varepsilon_{x x}\right)$, and the isotropic compression modulus $E=$ $\left(C_{1}+C_{2}+2 G\right) / 2$ that reacts to an isotropic deformation, and the anisotropic modulus $A=\left(C_{2}-\right.$ $\left.C_{1}\right) / 2$ that reacts to a deviatoric deformation. And for the deviatoric stress one has:

$$
\delta \sigma_{D}=\delta\left(\sigma_{z z}-\sigma_{x x}\right) / 2=E\left(\frac{A}{E} \varepsilon_{V}+\frac{E-2 G}{E} \varepsilon_{D}\right)+\delta \sigma_{D}^{\text {struct. }},
$$

with the anisotropic shear modulus $B=\left(C_{1}+C_{2}-2 G\right) / 2=E-2 G$ that reacts to an anisotropic deformation. Thus the anisotropic, elastic material behavior is described (in the biaxial cartesian coordinates) by the compression modulus $E$ and the two dimensionless numbers $A / E$ that quantifies anisotropy, and $B / E$ that quantifies biaxial shear strength. In the special case of an isotropic material $(A=0)$, one gets the standard linear (incremental) elastic behavior $\delta \sigma_{V}=E \varepsilon_{V}$ and $\delta \sigma_{D}=(E-2 G) \varepsilon_{D}$. For the more general anisotropic material, isotropic and deviatoric stresses are coupled to both isotropic and deviatoric deformations.

From the numerical simulations (data not shown here), the relations $E=\left(2 k / \pi g_{E}\right) F_{V}, G / E=$ $g_{G}=g_{G}^{n}+g_{G}^{t}$ and $A / E \approx f_{D}$ are evidenced, so that the above equations (22) and (23) can be rephrased in terms of the isotropic and deviatoric fabric components using the non dimensional stress $\sigma^{*}=\sigma\left(\pi g_{E} / 2 k\right)$ and disregarding the structural terms:

$$
\delta \sigma_{V}^{*}=F_{V}\left(\varepsilon_{V}+f_{D} \varepsilon_{D}\right) \text { and } \delta \sigma_{D}^{*}=F_{V}\left(f_{D} \varepsilon_{V}+\left(1-2 g_{G}\right) \varepsilon_{D}\right) .
$$

as recently proposed in similar form by (M. Lätzel, PhD-thesis, Univ. Stuttgart, 2003). Note that $g_{E} \approx 1.05 \pm 0.01$ is empirical, but does not change during the large deformations applied - and it is practically pressure independent. In contrast, $g_{G}^{n} \approx 0.254 \pm 0.001$ increases during shear up to a maximum of $0.274 \pm 0.001$, and reaches intermediate values in the critical state shear regime; however, it is also independent of $p$. The tangential contribution is here $g_{G}^{t} \sim-\mu\left(k^{t} / k\right) g_{G}^{n}$, so that one has $1-2 g_{G} \approx 1-\left(1-\mu k^{t} / k\right) / 2 \approx 0.55$ for the parameters used here. The relation $(A / E) \approx$ 
$f_{D}(1 \pm 0.01)$ is true in the initial deformation regime and becomes somewhat less reliable for larger deformations, corresponding to large $f_{D}$, and for larger $p$. Note that the tangential forces seemingly do not contribute to either $E$ or $A$, only $B$ is affected.

Note that the formulation of a more general constitutive law for arbitrary orientation of the deformation direction relative to the direction of the fabric eigen-values is far from the scope of this paper.

\section{Summary and Conclusion}

From the presented data, it can be concluded that there are basically only three different quantities in the stiffness tensor, scaling with the microscopic spring stiffness used for the simulation, which quantify the stress response of a static granular packing - disregarding changes of the structure.

All stress responses are proportional to the isotropic fabric $F_{V}$. The isotropic stress is proportional to the isotropic deformation and a compression modulus $E$, but also to the deviatoric deformation times the anisotropic modulus $A$, if the material is already anisotropic, as quantified by $f_{D}$. The anisotropic stress response is proportional to the biaxial shear modulus $B$ and the deviatoric deformation, but it is also proportional to the isotropic deformation times the anisotropic modulus $A$.

Furthermore, two simple constitutive relations for the evolution of the deviatoric fabric and stress with the deviatoric deformation are proposed. In both cases, the limit deviator magnitude is approached exponentially fast. The microscopic and structural reasons for the limits in anisotropy are unclear as well as the detailed relations between stress, strain, and anisotropy, especially in the presence of structural changes.

Acknowledgements

We thank E. Clément, J. Jenkins, N. P. Kruyt, M. Madadi, F. Nicot, R. G. Rojo, C. Thornton, J. Tomas, and R. Tykhoniuk for helpful discussions and acknowledge the support of the Deutsche Forschungsgemeinschaft (DFG). This work is part of the research programme of the Stichting voor Fundamenteel Onderzoek der Materie (FOM), financially supported by the Nederlandse Organisatie voor Wetenschappelijk Onderzoek (NWO) and the Stichting Shell Research.

\section{REFERENCES}

Babić, M. (1997). Average balance equations for granular materials. Int. J. Engng. Sci., 35(5):523-548.

Bagi, K. (1996). Stress and strain in granular assemblies. Mech. of Materials, 22:165-177.

Bagi, K. (1999). Microstructural stress tensor of granular assemblies with volume forces. $J$. Appl. Mech., 66:934-936.

Ball, R. C. and Blumenfeld, R. (2002). Stress field in granular systems: Loop forces and potential formulation. Phys. Rev. Lett., 88:115505.

Bardet, J. P. and Proubet, J. (1991). A numerical investigation of the structure of persistent shear bands in granular media. Géotechnique, 41(4):599-613.

Bardet, J. P. and Vardoulakis, I. (2001). The asymmetry of stress in granular media. Int. J. of Solids and Structures, 38(2):353-367.

Bashir, Y. M. and Goddard, J. D. (1991). A novel simulation method for the quasi-static mechanics of granular assemblages. J. Rheol., 35(5):849-885.

Bathurst, R. J. and Rothenburg, L. (1988). Micromechanical aspects of isotropic granular assemblies with linear contact interactions. J. Appl. Mech., 55:17-23.

Calvetti, F., Combe, G., and Lanier, J. (1997). Experimental micromechanical analysis of a 2d granular material: relation between structure evolution and loading path. Mech. Coh. Fric. Mat., 2:121-163.

Chang, C. S. and Ma, L. (1991). A micromechanical-based micropolar theory for deformation of granular solids. Int. J. of Solids and Structures, 21(1):67-86. 
Cundall, P. A. and Strack, O. D. L. (1979). A discrete numerical model for granular assemblies. Géotechnique, 29(1):47-65.

Goldhirsch, I. and Goldenberg, C. (2002). On the microscopic foundations of elasticity. Eur. Phys. J. E, 9(3):245-251.

Herrmann, H. J., Hovi, J.-P., and Luding, S., editors (1998). Physics of dry granular media NATO ASI Series E 350, Dordrecht. Kluwer Academic Publishers.

Kishino, Y., editor (2001). Powders \& Grains 2001, Rotterdam. Balkema.

Kruyt, N. P. (2003). Statics and kinematics of discrete cosserat-type granular materials. Int. J. of Solids and Structures, 40(3):511-534.

Kruyt, N. P. and Rothenburg, L. (1998). Statistical theories for the elastic moduli of twodimensional assemblies of granular materials. Int. J. of Engineering Science, 36:1127-2242.

Kruyt, N. P. and Rothenburg, L. (2001). Statistics of the elastic behavior of granular materials. Int. J. of Solids and Structures, 38:4879-4899.

Lätzel, M., Luding, S., and Herrmann, H. J. (2000). Macroscopic material properties from quasi-static, microscopic simulations of a two-dimensional shear-cell. Granular Matter, 2(3):123-135. cond-mat/0003180.

Liao, C.-L. and Chang, T.-C. (1997). A generalized constitutive relation for a randomly packed particle assembly. Computers and Geotechnics, 20(3/4):345-363.

Luding, S. (2004). Micro-macro transition for an-isotropic, frictional granular packings, Int. J. Sol. Struct. (in press).

Luding, S. (2004b). Micro-macro transition for anisotropic granular solids. (in preparation).

Luding, S. and Herrmann, H. J. (2001). Micro-macro transition for cohesive granular media. in: Bericht Nr. II-7, Inst. für Mechanik, Universität Stuttgart, S. Diebels (Ed.).

Luding, S., Tykhoniuk, R., and Tomas, J. (2003). Anisotropic material behavior in dense, cohesive powders. Chem. Eng. Technol., 26(12):1229-1232.

Madadi, M., Tsoungui, O., Lätzel, M., and Luding, S. (2004). On the fabric tensor of polydisperse granular media in 2d. Int. J. Sol. Struct., 41(9-10):2563-2580.

Oda, M. and Iwashita, K. (2000). Study on couple stress and shear band development in granular media based on numerical simulation analyses. Int. J. of Enginering Science, 38:17131740.

Peters, J. P. and Horner, D. A. (2002). Errors of scale in discrete element computations. In Cook, K. and Jensen, R. P., editors, Discrete Element Methods: Numerical Modeling of Discontinua, pages 56-67, Santa Fe. ASCE.

Rothenburg, L. and Selvadurai, A. P. S. (1981). A micromechanical definition of the Cauchy stress tensor for particulate media. In Selvadurai, A. P. S., editor, Mechanics of Structured Media, pages 469-486. Elsevier, Amsterdam.

Suiker, A. S. J., Metrikine, A. V., and de Borst, R. (2001). Comparions of wave propagation characterisitcs of the cosserat continuum model and corresponding discrete lattice models. Int. J. of Solids and Structures, 38:1563-1583.

Thornton, C. (2000). Numerical simulations of deviatoric shear deformation of granular media. Géotechnique, 50(1):43-53.

Thornton, C. and Antony, S. J. (2000). Quasi-static deformation of a soft particle system. Powder Technology, 109(1-3):179-191.

van Baars, S. (1996). Discrete Element Analysis of Granular Materials. $\mathrm{PhD}$ thesis, Technische Universiteit Delft, Delft, Nederlands.

Vermeer, P. A., Diebels, S., Ehlers, W., Herrmann, H. J., Luding, S., and Ramm, E., editors (2001). Continuous and Discontinuous Modelling of Cohesive Frictional Materials, Berlin. Springer. Lecture Notes in Physics 568. 